\title{
Reflets
}

Revue d'intervention sociale et communautaire

\section{Familles immigrantes et handicap de développement : une double minorisation?}

\section{Jerryne Mahele-Nyota}

Volume 16, numéro 2, 2010

URI : https://id.erudit.org/iderudit/1000320ar

DOI : https://doi.org/10.7202/1000320ar

Aller au sommaire du numéro

Éditeur(s)

Reflets, Revue d'intervention sociale et communautaire

ISSN

1203-4576 (imprimé)

1712-8498 (numérique)

Découvrir la revue

Citer cet article

Mahele-Nyota, J. (2010). Familles immigrantes et handicap de développement : une double minorisation? Reflets, 16(2), 214-234.

https://doi.org/10.7202/1000320ar d'utilisation que vous pouvez consulter en ligne.

https://apropos.erudit.org/fr/usagers/politique-dutilisation/ 


\section{Familles immigrantes et handicap de développement : une double minorisation?}

\section{Jerryne Mahele-Nyota}

Depuis fort longtemps, la société canadienne est façonnée par l'immigration. À leur arrivée au Canada, la plupart des familles immigrantes disposent de peu de connaissances sur le pays et sur son fonctionnement, et cela, malgré les diverses agences qui ont pour mission de les aider ou les organismes qui sont représentatifs de groupes ethniques et qui opèrent dans les grandes villes canadiennes. L'établissement au Canada est moins problématique pour les familles immigrantes possédant des caractéristiques culturelles, ethniques et religieuses - similaires à celles de la population dominante ici, de même que pour les familles pouvant maintenir un statut social et financier semblable à celui qu'elles avaient dans leur pays d'origine (Este, 1999, p. 19).

Pour les autres cependant, la vie peut être très difficile. Ces familles se heurtent à plusieurs obstacles : adaptation à un nouvel environnement linguistique, culturel, climatique, économique, ainsi qu'à un nouveau système social. La vie est encore plus difficile pour une famille qui arrive au Canada avec un enfant ayant un handicap de développement ou dont l'enfant reçoit ce diagnostic à son arrivée ici.Aux défis que représente sa condition d'immigrante s'ajoutent les responsabilités accrues envers cet enfant, lesquelles peuvent se compliquer à cause d'une perception du handicap souvent différente dans le pays d'accueil.

Confrontées à des difficultés, les familles devront faire appel aux services de nombreux organismes de la région où elles habitent. Comme ces services n'ont pas au départ été mis en 
place en tenant compte des spécificités et des contextes culturels des familles immigrantes, leur capacité de leur offrir des réponses adéquates est parfois limitée. La pénurie de recherche sur les difficultés rencontrées par les familles immigrantes ayant un enfant avec un handicap de développement nous a incitée à explorer cette problématique dans le cadre d'un mémoire de maîtrise. Dans cet article, nous jetterons un regard sur la manière dont le handicap est perçu dans certaines cultures et nous verrons comment les personnes immigrantes qui vivent avec un handicap, ainsi que leur famille, peuvent être doublement minorisées. Puis, nous mettrons en lumière certains des obstacles émanant des services qui contribuent à les exclure ou à les isoler. Des pistes de recommandation seront ensuite suggérées afin que les organismes soient en mesure d'améliorer leurs services dans le but de mieux répondre aux besoins d'une clientèle multiethnique.

\section{Variations dans l'univers de sens du handicap}

Brown (2003, p. 19) désigne le handicap de développement comme une condition de la personne qui l'empêche à travers le temps d'accomplir certaines choses qui sont attendues socialement. Selon Graziano (2002, p. 21), le handicap de développement peut se manifester sous différentes formes, entre autres, par des incapacités physiques, la maladie, des troubles sensoriels, des incapacités cognitives, des troubles émotionnels ou comportementaux. Jusqu'au début du $\mathrm{XX}^{\mathrm{e}}$ siècle, les causes du handicap de développement étaient considérées en Occident comme un mystère et il était souvent attribué aux démons, aux mauvais esprits ou à un péché commis par un parent. Le développement de la médecine a modifié cette perception; aujourd'hui, des facteurs multiples expliquent le handicap de développement et on reconnait qu'il n'existe pas d'étiologie unique (Brown, 2003).

Par ailleurs, bien que le handicap soit un fait universel, sa signification ne dépend pas seulement de sa nature biologique. En effet, le sens que l'on donne au handicap est influencé par 
les croyances et valeurs qui prédominent dans une société déterminée. Ainsi, entre les cultures et au sein même des cultures, il existe certaines variations considérables dans les interprétations, définitions et catégorisations du handicap (Ingstad et Reynolds Whyte, 1995, p. ix; Harris, 2004). Chaque société a sa propre interprétation du handicap et de son développement, de la manière de traiter les individus qui en sont atteints, des rôles qu'ils peuvent remplir et des droits dont ils peuvent ou non disposer (Groce, 1999).

Au Canada, le concept du handicap est modelé par les valeurs euro-américaines de l'égalité et de l'aptitude individuelle qui font partie de l'identité sociale (Ingstad et Reynolds Whyte, 1995). D'après ces dernières, l'univers de sens du handicap en Amérique du Nord est conçu à l'intérieur d'un système qui considère que la personne subissant un handicap est égale aux autres citoyens. De ce fait, on doit faire en sorte qu'elle soit une personne à part entière, donc traitée de manière équitable et surtout ayant les moyens de réussir et d'être autonome. L'élaboration de lois, de procédures administratives, de diagnostics médicaux, ainsi que la mise sur pied de services spécialisés ont pour but de favoriser l'autonomie individuelle de la personne ayant le handicap de développement (Ingstaad et Reynolds Whyte, 1995, p. 10). Le handicap devient ainsi un problème clinique, individuel, impliquant la personne qui vit avec le handicap ainsi que sa famille.

En Afrique subsaharienne, l'étiologie du handicap de développement est très diversifiée. Cependant, elle est presque toujours basée sur des relations problématiques que la personne ayant un handicap, ses parents ou les membres de sa famille entretiennent avec la société et le monde surnaturel.Ainsi, l'origine du handicap se rattache à l'interaction qui se fait entre le social et le surnaturel (Ingstad et Reynolds Whyte, 1995a, p. 37). De ce fait, l'idée de blâme se trouve liée au handicap. En effet, on aura tendance à couvrir d'opprobre l'individu qui nait avec un handicap ou devient handicapé à la suite de circonstances particulières. Cette attitude peut varier selon la région, selon le statut de la personne souffrant du handicap ou encore selon son entourage (Ingstad, 1990; Reynolds Whyte, 1995, p. 273). Chercher la cause du handicap 
de développement, c'est en général déterminer si l'individu ou les gens qui l'entourent sont responsables de sa condition. Un lien s'établit ainsi entre la causalité du handicap et l'individu ou son entourage.

Le handicap est perçu alors comme "le signe d'un désordre social ou cosmique qui se projette sur le corps humain " (Ingstad et Reynolds Whyte, c1995a , p. 37). Cette perception s'oppose à l'explication strictement biologique du modèle occidental; on n'y répondra pas selon le modèle médical, mais selon un modèle sociétal (Reynolds Whyte, 1995, p. 274).

En revanche, le blâme et la stigmatisation restent souvent les mêmes, peu importe le lieu ou la culture. En effet, certains événements récents au Canada illustrent bien une tendance à se demander si la mère d'un enfant handicapé a bu, fumé ou pris de la drogue durant sa grossesse. Même si l'univers de sens du handicap varie selon les cultures, la stigmatisation est d'une certaine manière toujours présente (Groce, 2005, p. 7).

\section{Le handicap place la personne et les familles immigrantes dans une situation doublement minoritaire}

Que ce soit dans une société où le handicap est davantage perçu comme un attribut individuel ou dans une autre qui est axée sur la collectivité et où il est perçu comme un problème personnel, familial ou même ancestral, en relation avec son environnement social, naturel et surnaturel, cette condition confere automatiquement à la personne ou à sa famille une appartenance à un groupe minoritaire.

Comme le soutient Dorvil (2001,p. 197), lorsque le handicap est présenté comme une caractéristique individuelle dévalorisante découlant automatiquement d'une incapacité ou d'une déficience, on tient pour acquis que l'individu est nécessairement inférieur, mal placé, hors concours, disqualifié. Cet individu « fait pitié » avec 
sa différence; son handicap devient une particularité sur laquelle on va se concentrer, le transformant par le fait même en victime.

Dans les cultures où le sens du handicap se construit autour des rapports sociaux - familiaux ou communautaires — et où le handicap de développement devient le reflet de la faute commise par un individu ou une famille en relation avec l'ordre social et surnaturel, les connotations négatives dont il est porteur peuvent amener la famille à cacher sa condition. Ainsi, la famille immigrante ou réfugiée qui arrive au Canada et qui perçoit le handicap comme une conséquence de relations problématiques avec la société et le monde surnaturel va d'elle-même avoir comme première réaction de s'exclure et de s'isoler, de ne pas aller chercher les services disponibles, se plaçant de ce fait en situation doublement minoritaire.

Doublement minoritaire à cause du handicap et du statut d'immigré. Comme le souligne Jaqueline Costa-Lascoux (1996), le terme "immigré » est porteur de stéréotypes qui marquent la distance sociale et la différence culturelle. La relation d'altérité est entendue comme une relation inégale à la société d'accueil, majoritaire et dominante. Ainsi, par le jeu des représentations, les immigrés apparaissent à plusieurs titres parmi les " exclus". Et pour complexifier davantage la situation de ces familles, la manière dont les services sont mis en place aura aussi tendance à les placer en situation d'exclusion en ne tenant pas compte de leurs caractéristiques culturelles.

\section{Contexte de la recherche}

Dans le cadre d'une recherche de type exploratoire nous avons effectué des entrevues semi-dirigées auprès de huit intervenants travaillant au sein d'organismes de la ville d'Ottawa qui offrent des services aux familles immigrantes et réfugiées ayant un enfant avec un handicap de développement. Cela, afin de mieux connaître la façon d'opérer de ces organismes, les moyens qu'ils offrent à ces familles pour qu'elles aient accès à leurs services ainsi que les obstacles rencontrés. 
Les participants devaient être des responsables d'organismes d'Ottawa, anglophones ou francophones, offrant des services aux enfants atteints d'un handicap de développement ainsi qu'à leurs familles. Bien qu'il existe divers types d'organismes, nous avons sélectionné ceux auxquels les familles faisaient souvent appel à cause de la spécificité de leurs services et selon les besoins exprimés par ces dernières.

Les questions d'entrevue ont été élaborées à partir de lectures sur la compétence culturelle des organismes. De façon plus précise, les questions suivantes nous ont guidée : Quels sont les obstacles rencontrés par les familles qui ont un enfant avec un handicap de développement? Quels sont les mécanismes mis en place pour adapter les services aux besoins de cette clientèle? Quels sont les obstacles empêchant l'adaptation des services? De quoi les organismes auraient-ils besoin pour poursuivre dans cette voie et quels sont leurs souhaits?

\section{Facteurs ayant un impact sur l'accès aux services}

Les familles immigrantes ayant un enfant avec un handicap sont confrontées à des obstacles qui nuisent à leur intégration et qui sont autant de facteurs d'intensification de leur état de minoritaires. Notre étude a permis d'en identifier plusieurs.

Nous traiterons ici des facteurs structurels, de ceux émanant du type de services offerts et du manque de sensibilisation de la part des intervenants.

\section{Facteurs structurels}

Parmi les divers facteurs structurels affectant l'accès des familles aux services, les intervenants soulignent celui de la carte santé. En effet, sans cette carte de nombreuses agences ne peuvent fournir de services à l'enfant atteint de handicap de développement et cela, même si l'enfant requiert des soins importants. Dans ce cas, il est possible de référer la famille à des services privés. Cette constatation rejoint celle de Statistique Canada (2003) qui souligne que le fait de ne pas posséder la carte permettant 
d'accéder aux services de santé constitue l'une des principales difficultés qu'éprouvent les nouveaux arrivants dont le statut n'est pas encore régularisé.

Des intervenantes signalent aussi que plusieurs des familles qu'elles rencontrent ont des difficultés financières, ce qui peut avoir un impact sur l'accès aux services. Les familles immigrantes font en effet partie de la catégorie des personnes dont les ressources financières sont les plus faibles (Statistique Canada, 2003). En dépit d'un niveau d'éducation souvent supérieur à celui des Canadiens de souche, les personnes immigrantes sont relativement pauvres comparativement à la population majoritaire. Cela s'explique notamment par des pratiques d'employeurs et des politiques d'ordre professionnel qui ne reconnaissent généralement ni les diplômes ni les expériences de travail non canadiennes des immigrants (Levesque, 2005). Cette situation place ces derniers en situation financière précaire et les rend plus susceptibles de dépendre de l'aide sociale.

\section{Facteurs liés à l'organisation et aux modèles de service}

L'organisation des services est conçue de telle manière qu'elle peut constituer un obstacle pour les familles immigrantes. Il existe une multitude de services destinés aux enfants ayant un handicap de développement et chaque organisme fonctionne d'une manière qui lui est propre, ce qui est souvent difficile à comprendre ou à décoder pour les familles immigrantes devant se familiariser avec les complexités administratives entourant les services.

Les répondants expliquent que la fragmentation des services entraîne des difficultés et des incompréhensions pour les familles qui ont du mal à s'y retrouver et qui, pour accéder aux ressources nécessaires, ont besoin de l'aide d'une travailleuse sociale afin de comprendre le fonctionnement du système et affronter la bureaucratie. Il faudrait donc trouver une façon de supprimer la lourdeur administrative de plusieurs organismes, car cela constitue sans aucun doute un obstacle pour ces familles. Anderson et collab. (1990, p. 252) soutiennent d'ailleurs que les familles ont besoin d'être conseillées sur la manière dont le système fonctionne au 
Canada, non seulement parce qu'il est différent du système de leur pays d'origine, mais aussi parce qu'il est complexe et souvent bureaucratique.

Certaines intervenantes ont formulé des préoccupations face aux tests d'évaluation qui peuvent, à leur avis, être biaisés, car ils ne tiennent pas compte de la culture d'origine des enfants à qui ils sont administrés. Plusieurs auteurs (Ionescu, 1996; Sedighdeilami et Gindi, 2003) ont formulé ces mêmes inquiétudes. Ils ont critiqué les instruments d'évaluation utilisés en Ontario, dont la plupart ont été développés et validés aux États-Unis et élaborés selon une perspective occidentale ou eurocentrique (Sedighdeilami et Gindi, 2003, p. 485). D'après ces mêmes auteurs, les résultats des tests pourraient être biaisés, entraîner une mauvaise compréhension des aptitudes et conduire à un diagnostic de retard mental chez un grand nombre des enfants issus de groupes ethnoculturels et marginalisés. Cette situation soulève des problèmes pour quelques intervenantes qui ont remarqué un grand nombre d'enfants immigrants au sein des classes spécialisées.

Quelques participantes ont expliqué que les approches behavioristes axées sur la modification de comportement largement utilisées dans les services peuvent ne pas convenir aux familles ou s'avérer problématiques, et cela, pour plusieurs raisons. Ce modèle met l'accent sur les comportements problématiques qu'il faut modifier après évaluation systématique. Par exemple, si un enfant est persuadé qu'il n'obtient l'attention des parents qu'en faisant des crises de colère jusqu'à se blesser, il aura tendance à les répéter, même si l'attention qu'il reçoit est négative. Si la solution préconisée est que les parents évitent de prêter attention à ce genre de comportement, il peut être difficile pour beaucoup d'ignorer le comportement "automutilant " de l'enfant, notamment s'il ravive des souvenirs pénibles, surtout chez les familles réfugiées. Aussi, la technique peut être assez exigeante étant donné qu'elle nécessite une mesure et un enregistrement continus et minutieux des comportements observés de l'enfant.

Par ailleurs, nombre d'interventions éducatives peuvent sembler étranges aux familles immigrantes, surtout qu'en Amérique du Nord on encourage l'autonomie de l'enfant dès sa naissance 
(Lynch et Hanson, 2004, p. 11). Comme les intervenantes voient leurs interventions à travers le prisme de la culture occidentale, elles jugent ces méthodes absolument nécessaires au bien-être de l'enfant et ont de la difficulté à travailler avec des familles qui n'y adhèrent pas. La plupart des parents pourraient se sentir dépassés devant la multitude de méthodes d'intervention proposées, car elles different de leurs méthodes d'éducation parentale. Cela entraîne chez les parents un sentiment d'incompétence qu'ils ont de la difficulté à accepter, ayant l'impression de faillir à leur rôle parental.

Notre recherche a aussi permis de constater que les modèles d'intervention auprès des familles varient d'un organisme à un autre. Certains perçoivent la famille comme un ensemble et donc préconisent l'approche systémique familiale. Pour d'autres organismes, seuls les besoins de l'enfant sont pris en considération. Or les facteurs familiaux sont importants pour les familles immigrantes ou réfugiées. Par exemple, si les parents connaissent des difficultés financières ou se rapportant à leur statut d'immigration, ces problèmes vont automatiquement avoir un impact sur toute la famille et sur l'enfant handicapé. C'est la raison pour laquelle il est impossible d'intervenir auprès de l'enfant handicapé en faisant abstraction de son environnement; d'où la nécessité d'effectuer un examen critique de l'ensemble du contexte familial dans lequel évolue l'enfant vivant un handicap de développement (Harris, 2004, p. 32).

\section{Difficultés de communication et manque de sensibilisation et de connaissances chez les intervenants}

Les répondantes identifient les problèmes de communication avec les familles comme étant des plus importants. Ils peuvent être causés par une incompréhension de la langue qui nécessiterait des services de traduction ou d'interprétation. Cette barrière de la langue ou la conscience d'avoir un accent différent peuvent avoir un impact considérable sur la participation des parents au sein de certains programmes ou sur l'accès des familles immigrantes à certains services (Doyle et Visano, 1987b, p. 17). D'où la 
nécessité de requérir aux services d'interprètes et de traducteurs compétents.

Quelques organismes ont mis en place un système permettant d'évaluer chez les familles la compréhension du français ou de l'anglais. Certains organismes cherchent à connaitre également la langue parlée à la maison. D'autres enfin s'attendent à ce que les familles fassent part d'elles-mêmes de leurs besoins de communication.

Afin de fournir un meilleur service, il est important que l'identification linguistique se fasse au sein des services d'accueil. Divers systèmes d'identification permettent de savoir si les familles ont besoin d'un service d'interprétariat ou de traduction. D'ailleurs, comme le précisent Burwell et Day (1996, p. 59), intervenir auprès des familles parlant une langue différente peut souvent causer des problèmes, d'où la nécessité d'avoir un interprète pour faciliter la communication. Mais afin d'éviter la modification ou la perte de la qualité de l'information, il est primordial de recourir à des interprètes compétents et en qui l'on peut avoir confiance (Sedighdeilami et Gindi (2003, p. 484). Il est tout aussi important d'avoir un interprète dont la culture correspondrait à celle des familles afin d'éviter des problèmes d'éthique (Harris, 2004, p. 49). De plus, dans le choix de l'interprète, il est nécessaire de tenir compte du genre afin d'éviter certains problèmes.

Cependant, notre recherche a permis de constater que la plupart des organismes ne possédent pas de budget pour ce genre de service. Une répondante nous a confié que quelques agences ignorent les besoins linguistiques des familles. Il en résulte que d'autres agences doivent pallier cette lacune. Une autre répondante a déploré l'absence de coordination interagence concernant le recours à un interprète. Elle suggère que les agences se partagent les frais. Malheureusement, ces dernières ne sont pas en mesure de fournir les services d'interprétation dans toutes les langues; c'est pour cela qu'elles acceptent que les familles aient recours à des personnes de leur entourage pour mieux se faire comprendre.

Concernant l'interprétation culturelle, très peu d'intervenantes interrogées nous ont fait part de l'utilisation de ce 
genre de service. Selon Massé (1995), le problème d'accès aux services de santé ne se résume pas à un simple problème de traduction ou d'interprétation. En fait, le véritable défi demeure dans l'interprétation culturelle, c'est-à-dire dans le contexte de notre étude, dans la compréhension de la signification attribuée aux handicaps de développement d'une culture à l'autre. Mais l'interprétation culturelle réside aussi dans l'identification des codes culturels qui peuvent survenir dans l'interaction entre famille et intervenantes, codes qui different souvent d'une culture à l'autre. L'interprétation culturelle pourrait faciliter la relation avec les familles et permettre de traduire et faire comprendre leurs univers de sens aux intervenants.

Le manque de connaissance du vécu et de la culture des familles est un autre facteur qui constitue un obstacle pour les intervenantes interrogées. Elles disposent de peu de connaissances formelles concernant les diverses normes culturelles et avouent par conséquent ne se fier qu'à leur sens de l'observation.

Selon Sedighdeilami et Gindi (2003, p. 480), ne pas se familiariser avec certaines normes culturelles et ne pas tenir compte des différences culturelles durant l'intervention pourrait entrâner son échec. Car chaque culture possède un style de communication qui lui est propre et qui donne une signification particulière à la communication (Sedighdeilami et Gindi, 2003, p. 482). Pour Cohen-Emerique (1989), les difficultés que les intervenants pourraient rencontrer avec les familles seraient dues à une méconnaissance de leur culture. Il faudrait que les organismes favorisent la connaissance des diverses cultures, car connaitre les familles dans toutes leurs dimensions - psychologique, culturelle, sociale et historique - est fondamental dans le processus d'aide (Cohen-Emerique, 1989; Grant, 1994).

Cohen-Emerique (1989) précise par ailleurs qu'il ne faudrait pas se limiter à la connaissance des spécificités culturelles, car la culture d'origine est une notion trop large : on peut trouver une diversité d'appartenances chez les habitants d'un même pays d'origine. De plus, il ne faut pas oublier que l'individu, selon son pays d'origine ou son lieu de vie, a déjà été en contact de façon plus ou moins significative avec la culture occidentale. Il a donc 
déjà connu un processus d'acculturation. Cela est plus prégnant chez les familles originaires de pays autrefois colonisés.

De surcroit, lorsqu'une personne immigre, elle doit s'adapter au nouveau pays, ainsi qu'à ses us et coutumes. Ainsi, beaucoup d'immigrants doivent changer leurs habitudes alimentaires, s'adapter à une nouvelle culture, à une nouvelle langue et à un nouveau climat. Il leur faut également trouver de nouveaux soutiens sociaux, faire face au racisme et à la discrimination. Or, tous ces éléments liés au processus d'intégration ont un impact considérable sur la santé des immigrants (Legault, 2000; Levesque, 2005, Waxler-Morrison, 1990, p. 7). En effet, l'arrivée et l'installation dans un nouveau pays occasionnent souvent un stress important (Conseil canadien de la santé multiculturelle, 1989, dans Llene Hyman, 2001).

Plusieurs enquêtes et analyses confirment que les situations de stress reliées au processus migratoire ont effectivement une incidence sur la santé mentale des réfugiés (Tobin et Friedman, 1983, dans Legault, 2000; Potocky-Tripodi, 2002). En dépit de l'importance de ces facteurs sur la situation des familles, nos entretiens nous ont permis de constater que certaines répondantes semblent ignorer les conditions d'immigration des familles. Parfois, elles affirment clairement ne pas avoir connaissance des problèmes d'intégration vécus par les familles. Par contre, d'autres intervenantes constatent combien les conditions de l'immigration sont autant de facteurs de stress.

Notre étude a aussi permis de constater que peu d'intervenantes possèdent des notions sur les lois d'immigration. Certaines ne voient pas de lien entre les services qu'elles rendent aux familles et le fait de posséder des connaissances sur les lois et les règlements de l'immigration. D'autres disent qu'elles ne voient pas l'utilité d'en connaitre davantage.

Anderson et collab. (1990, p. 247) soutiennent qu'il est primordial pour les intervenants d'être au courant des difficultés rencontrées par les familles immigrantes qui décident de s'établir dans un nouveau pays. Dans une recherche effectuée par Doyle etVisano (1987a, p. 12), la critique la plus fréquente à l'égard des 
intervenants concerne leur incapacité à connaittre les situations difficiles vécues par les nouveaux arrivants.

\section{Pistes de réflexion pour les intervenants et les organismes}

\section{Promotion et information}

Selon Harris (2004, p. 73), la promotion des services exercée auprès des communautés ethnoculturelles doit être culturellement compétente. Il ajoute par ailleurs que l'information que véhiculent brochures et dépliants a souvent été développée sans tenir compte des groupes ethnoculturels. Il est donc essentiel que, traduite, cette information soit rendue accessible et pertinente pour les différents groupes culturels. C'est pour cela que les images et le langage utilisés dans le matériel d'information doivent être aussi culturellement appropriés.

Pourtant, d'après Harris (2004, p. 73) et Masi (1993, p.15), la promotion des services ne se limite pas au matériel d'information. Selon eux, pour augmenter l'accès à leurs services, les organismes doivent créer un environnement physique accueillant, à l'aide d'images, d'affiches et de matériel reflétant l'origine culturelle des familles qu'ils desservent.

Selon Harris (2004, p. 70), l'outreach est par rapport à la problématique du handicap l'un des éléments essentiels de la compétence culturelle. Il permet de rejoindre les personnes issues de différents groupes culturels pour assurer leur pleine participation ainsi que des opportunités égales au sein des programmes et services. C'est une stratégie essentielle dont le but est d'organiser la participation de la communauté et d'informer les groupes culturels sur les divers services existants. En ce qui concerne la problématique du handicap, Harris suggère plusieurs stratégies d'outreach permettant d'établir des liens avec divers groupes :

1. déployer des efforts de promotion auprès des communautés ethniques dans le but d'identifier et d'aborder les besoins non comblés des groupes peu ou pas desservis faute d'outreach; 
2. utiliser des méthodes d'outreach non traditionnelles afin d'informer sur les services existants les communautés et les groupes ethniques mal desservis. Il est possible, par exemple, d'effectuer des présentations informelles auprès des groupes communautaires durant des périodes bien spécifiques, comme lors des festivités;

3. organiser des groupes de discussion afin d'identifier les besoins des familles et de les incorporer dans les programmes à venir.

Si pour diverses raisons l'outreach n'est pas envisageable, la collaboration avec les différents groupes communautaires pourrait servir de moyen de remplacement.

\section{Collaboration avec les différents groupes communautaires}

D'après Masi (1993, p. 25), pour satisfaire les besoins des familles ethnoculturelles, les services et les programmes doivent être soutenus par la communauté dans laquelle vivent ces dernières. Ce soutien de la communauté doit s'effectuer au tout début de l'implantation des programmes ou services, en incluant par exemple un représentant de la communauté au sein de la gouvernance de l'organisme (Masi, 1993, p. 24). Selon Kérisit (1994), afin de faciliter la participation des familles et des membres des communautés ethnoculturelles dans la planification, l'amélioration et le développement des services, il faut informer les communautés de l'existence de ces services. Cela peut se faire en informant les membres des communautés ethnoculturelles sur la façon de s'impliquer dans le développement et l'amélioration des services.

Cela peut également se faire en facilitant la communication avec les familles ethnoculturelles et en développant des mécanismes permettant d'examiner les politiques et pratiques liées à l'accès aux services des membres de minorités ethniques. D'après Harris $(2004$, p. 67), la collaboration avec des communautés ethnoculturelles est l'élément clé pour la résolution de plusieurs problèmes. De plus, elle peut fournir des mécanismes essentiels à la compréhension des différentes perceptions du handicap et faciliter l'accès des familles aux services. 
Cette collaboration ne devrait pas seulement se limiter aux différents groupes ethniques. Elle peut aussi se faire avec des agences fournissant des services aux immigrants et réfugiés. Thompson (1993, p. 219) qualifie cette collaboration d'extrêmement importante vu que ces agences sont informées des besoins et des problèmes des familles. Elles peuvent aussi servir de lien avec les communautés multiculturelles. La mise en place d'un système d'identification de l'origine culturelle des familles pourrait donc faciliter la tâche aux organismes.

\section{Identification de 1'origine culturelle des familles}

Selon le Department of Human Services de Victoria (Australie) (2004), un système d'identification permettrait de récolter l'information sur le pays d'origine de la famille, la langue parlée à la maison et les compétences dans la langue - ou les langues — du pays d'accueil.

L'absence d'identification de l'origine culturelle des familles constitue, d'après certains auteurs, un obstacle considérable. En effet, selon Doyle et Visano (1987b, p. 117), les organismes doivent développer un système d'identification permettant de réunir des données sur les caractéristiques des groupes culturels, afin d'améliorer la planification des services, leur livraison et leur accessibilité. Rassembler ce genre d'information est essentiel. D'après le guide du Department of Human Services de Victoria (Australie) (2004), cela permettrait d'évaluer une surreprésentation ou une sous-représentation d'un groupe en particulier et d'améliorer les services. Cela mènerait aussi au développement de nouvelles stratégies et modes d'intervention adaptés à certains groupes. Cependant, cette pratique doit être mise à jour régulièrement parce que les caractéristiques de la population peuvent changer et les besoins de services varier d'un groupe à un autre (Victorian Government Department of Human Services, 2004).

On peut se demander si le fait de recueillir de l'information sur l'origine culturelle des familles ne contrevient pas au respect de la vie privée. Cette interprétation est possible, car cela risque 
d'étiqueter certains groupes culturels. Pourtant, d'après Doyle et Visano (1987a, p. 16), cette interprétation des risques liés à cette pratique est exagérée et peut nuire à l'amélioration des services. Ces mêmes auteurs soutiennent que d'après la Commission ontarienne des droits de la personne, collecter des données culturelles ne constitue pas une infraction au code des droits de la personne si cette action a pour but de répondre aux besoins d'une clientèle et de faciliter la planification et la livraison des services à son égard. Cependant, cette pratique présuppose une formation appropriée.

\section{Développement de la compétence culturelle}

Recevoir des formations sur ce thème et sur la perception du handicap au sein de diverses cultures serait des plus utiles pour les intervenants. Ces formations permettraient d'en connaitre davantage sur la réalité des familles immigrantes et sur l'impact que le handicap peut avoir sur elles. Pour devenir culturellement compétent, un organisme doit exiger ce genre de formation. Une telle orientation indiquerait qu'il valorise et promeut la compétence culturelle et qu'il s'engage à la respecter (Lie et Este, 1999, p. 42).

Cependant, on ne doit pas se limiter à la formation sur la compétence culturelle. Une bonne représentativité des groupes ethnoculturels au sein de l'organisme constitue un élément essentiel à la compétence culturelle. Selon Harris (2004, p. 50), la présence de membres de divers groupes au sein du personnel offre plusieurs avantages, dont celui de mettre les familles à l'aise. Néanmoins, ces personnes doivent aussi être formées sur la communication interculturelle, car ce n'est pas parce qu'une intervenante est de la même origine culturelle que la famille que la communication sera facilitée. Le même auteur ajoute qu'une intervenante d'origine ethnique ne devrait pas être systématiquement responsable des familles des groupes culturels; cela en vue d'éviter un genre de "ghettoïsation» au sein de l'organisme.

D'après Doyle et Visano (1987a, p. 18), pour que l'organisme puisse être le reflet de la population immigrante, il faut mettre 
en place quelques mesures spécifiques. Il convient par exemple d'augmenter la représentation de divers groupes au sein de la direction et des postes de gestion et d'embaucher du personnel issu de divers groupes ethnoculturels, polyglotte et possédant des connaissances sur les communautés ethnoculturelles et de l'expérience auprès d'elles. Masi (1993, p. 24) ajoute qu'il faut favoriser la participation et la représentation de divers groupes culturels dès l'élaboration des programmes et services, et durant tout le processus de leur implantation.

Notre étude démontre qu'il y a peu de représentants des groupes ethnoculturels au sein de certains conseils d'administration. Pourtant, impliquer des membres des groupes ethnoculturels dans la gouvernance des organismes est essentiel à la compétence culturelle (Cultural Diversity Institute, 2000; Victorian Government Department of Human Services, 2004). D'où la nécessité pour eux d'encourager la nomination des membres des groupes ethnoculturels et de développer des politiques internes en ce sens.

Pour améliorer et faciliter l'accès des membres des groupes minoritaires aux services, la compétence culturelle doit être intégrée au sein de toute la culture organisationnelle. Ainsi, pour une meilleure accessibilité des services, plusieurs domaines doivent être abordés et examinés dans une perspective de changement organisationnel et dans le but d'éliminer les barrières visibles et invisibles d'une participation sociale complète des groupes non majoritaires (Thomas, 1987; Legault et Fortin, 1996). D’après Cross et collab. (1989) et Harris (2004), la compétence culturelle d'un système, d'un individu ou d'un organisme ne peut être effective à la suite d'une simple journée de formation, d'une consultation auprès d'experts, d'une lecture de manuel ou d'un cours. Bien au contraire, il s'agit véritablement d'un processus progressif et continuel d'acquisition de connaissances, de développement de compétences nouvelles ou améliorées et d'autoévaluation.

Ce processus permet par la suite d'atteindre le stade ultime du continuum de la compétence culturelle qui consiste à posséder " la capacité d’être culturellement compétent ». Cela signifie 
détenir toutes les attitudes, pratiques et politiques permettant de tenir compte des différences culturelles et de la diversité, être en mesure d'améliorer les services en observant et en partageant les recherches effectuées sur le champ de la compétence culturelle et en encourageant les relations de divers groupes culturels (Lum, 2003, p. 10).

Pour que sa mise en place soit réalisée avec succès, la compétence culturelle doit être appuyée et encouragée par tout le système, tant la structure de l'organisme que ses programmes et son personnel. Chacun doit agir comme un agent de changement, et cela à tous les niveaux (Harris, 2004, p. 5). Mais pour que l'application de cette notion soit totale et intégrée au sein de l'organisation, elle doit nécessairement faire partie des priorités stratégiques, car le chemin de la compétence culturelle requiert une planification circonspecte (Nash et Velazquez, 2003). En outre, comme la société canadienne ne cesse de se transformer aux niveaux national, régional et local, les organismes de services sociaux et de santé doivent percevoir la compétence culturelle non pas comme une option, mais plutôt comme une exigence de bon fonctionnement de leur part.

\section{Conclusion}

La réflexion sur l'analyse des données de notre étude nous amène à conclure qu'il serait utile de créer un centre indépendant destiné aux familles immigrantes et réfugiées ayant un enfant ou un autre membre de la famille avec un handicap de développement. Ce centre aurait plusieurs responsabilités, dont la principale serait de défendre les droits des familles; à Ottawa, il n'existe à présent aucune instance s'adressant à cette question. Ce centre offrirait aux personnes handicapées de différentes cultures ainsi qu'à leurs familles des ressources facilitant leur intégration. Créé sur le modèle de l'Association multiethnique pour l'intégration des personnes handicapées de Montréal ou de l'Ethno Racial People with Disabilities Coalition de Toronto, il aurait un rôle 
de diffusion des divers enjeux touchant les familles immigrantes, telle la conception différente du handicap.

Le centre pourrait se charger d'informer les familles sur le fonctionnement du système et leur faire prendre conscience de leur droit de regard sur la qualité des services qui leur sont destinés. Il se chargerait aussi de référer les familles aux services existants, d'offrir de la formation aux fournisseurs de service, aux organismes communautaires et aux ministères afin de leur permettre de travailler de manière efficace avec les familles.

Ce centre pourrait créer un contexte dans lequel les parents d'enfants ayant un handicap de développement seraient amenés à participer à la gouvernance des services. De plus, grâce à ce pôle d'information, les responsables des services seraient plus à même de créer des programmes culturellement compétents. Enfin, par le biais d'ateliers ou de séminaires, ce centre offrirait de la consultation aux intervenants d'autres institutions de santé, de services sociaux et d'organisations communautaires, ainsi qu'à un public plus large.

\section{Bibliographie}

ANDERSON, Joan M., et collab. (1990). "Cross-cultural caring : A handbook for health professionals ", dans Delivering culturally Sensitive Health Care, Nancy WAXLER-MORRISON, Joan ANDERSON et Elisabeth RICHARDSON (Dir.), Vancouver, UBC Press, p. 245-267.

BARNWELL, Deirdre, et Monimalika DAY (1996). " Providing support to diverse families ", dans Strategies for working with families of young children with disabilities, Paula J. BECKMAN (Dir.), Baltimore, Paul H. Brookes Publishing Co., p. 47-68.

BROWN, Ivan (2003). "What Do We Mean by Developmental Disabilities in Ontario? ", dans Developmental disabilities in Ontario, $2^{\mathrm{e}}$ éd., Ivan BROWN et Maire PERCY (Dir.), Ontario Association on Developmental Disabilities, p. 19-30.

COHEN-EMERIQUE, Margaret (1989). "Travailleurs sociaux et migrants : la reconnaissance identitaire dans le processus d'aide ", dans Choc de cultures C. CAMILLERI et M. COHENEMERIQUE (Dir.), Paris, L'Harmattan, p.77-115.

COSTA-LASCOUX, Jacqueline (1996). "Immigration : de l'exil à l'exclusion sociale ", dans L'exclusion, l'état des savoirs, Serge Paugam (Dir.), La Découverte, p. 158-170.

CROSS, Terry L., et collab. (1989). Towards a culturally competent system of care, Washington, DC, Georgetown University Child Development Center. 
DORVIL, Henri (2001). «Le handicap - Origines et actualité d'un concept », dans Problèmes sociaux (Tome I) - Théories et méthodologies, Henri Dorvil et Robert Mayer (Dir.), Presse de l'université du Québec, p. 191-215.

DOYLE, Robert, et LivyVISANO (1987a). A time for action: Access to health and social services for members of diverse cultural and racial groups, Toronto, Social Planning Council of Metropolitan Toronto.

DOYLE, Robert, et Livy VISANO (1987b). A program for action: Access to health and social services for members of diverse cultural and racial groups, Toronto, Social Planning Council of Metropolitan Toronto.

ESTE, David (1999). «Immigration, Multiculturalism and Social Welfare in Canada », dans Professional Social Service Delivery in a Multicultural World, Gwat-Yong LIE et David ESTE (Dir.), Toronto, Canadian Scholars' Press, p. 3-25.

GRANT, Robert (1994). The Symposium on persons with developmental delay in Ethnocultural Communities (January 2, 1994), Multiculturalism and Citizenship - Heritage Canada.

GRAZIANO, Anthony M. (2002). "Defining Developmental Disabilities ", dans Developmental disabilities : Introduction to a diverse field, Boston, Allyn et Bacon, p. 1-38.

GROCE, Nora (2005). "Immigrants, Disability, and Rehabilitation ", dans Culture and Disability, John H. STONE (Dir.), Thousand Oaks, Sage Publications, p. 1-14.

HARRIS, Patrick (2004). Culturally Competent Disability Support : Putting it into practice - A Review of the International and Australian Literature on Cultural Competence, Multicultural Disability Advocacy Association of New South Wales.

HANSON, Marci L., et Eleanor W. LYNCH (2004). Understanding families, Approaches to diversity, disability and risk, Baltimore, Paul $\mathrm{H}$. Brookes.

HYMAN, Llene (2001). Immigration et santé, Santé Canada.

INGSTAD, Benedicte (1990). «The Disabled Person in the Community : Social and Cultural Aspects ", International Journal of Rehabilitation Research, Vol. 13, No3, p. 187-194.

INGSTAD, Benedicte, et Susan REYNOLDS WHYTE (1995). "Disability and Culture : An Overview ", dans Disability and Culture, B. INGSTAD et S. REYNOLDS WHYTE (Dir.), Londres, California Press, p. ix-32.

INGSTAD, Benedicte, et Susan REYNOLDS WHYTE (1995a). "Disability, Cosmology, and Personhood ", dans Disability and Culture, B. INGSTAD et S. REYNOLDS WHYTE (Dir.), Londres, California Press, Partie I, p. 35-134.

IONESCU, Serban (1996). "L'approche interculturelle du retard mental : recherches et pratiques », dans Approches interculturelles en déficience mentale, Michel MERCIER, Serban IONESCU et Roger SALBREUX (Dir.), Namur, Presses Universitaires de Namur, p. 11 -28.

KÉRISIT, Michèle (1994). The Multicultural Bridging Project for individual with special needs and their families - October 1994, Steering Committee of the Multicultural Bridging Project.

LEGAULT, Gisèle, et Sylvie FORTIN (1996). "Problèmes sociaux et culturels des familles d'immigration récente. Perceptions des familles et des intervenants ", Actes du troisième symposium de recherches sur la famille : comprendre la famille, Sainte-Foy, Presses de l’Université du Québec.

LEGAULT, Gisèle (2000). "Parcours des réfugiés et des revendicateurs du statut de réfugié ", dans L'intervention interculturelle, Gisèle LEGAULT (Dir.), Boucherville, Gaëtan Morin, p. 109-127.

LEVESQUE, Annabel (2005). Les immigrants francophones en milieu minoritaire-services, défis, obstacles, Collège universitaire de Saint-Boniface. 
LIE, Gwat-Yong, et David ESTE (1999). Professional Social Service Delivery in a multicultural world, Toronto, Canadian Scholars' Press.

LUM, Doman (2003). Culturally competent practice, A framework for understanding diverse groups and justice issues, Brooks/Cole.

LYNCH, Eleanor W., et Marci L. HANSON (2004). Developing Cross-Cultural Competence, A guide for working with children and their families, Third Edition, Baltimore, Paul H. Brooks.

MASI, Ralph (1993). "Multicultural Health : Principles and Policies ", dans Health and CulturesExploring the relationships, Policies, Professional Practice and Education, Ralph MASI, Lynette MENSAH et Keith A. McLEOD (Dir.), Oakville, Ont., Mosaic Press,Vol.1, p. 11-31.

MASSÉ, Raymond (1995). Culture et santé publique, Québec, Gaëtan Morin.

NASH, Kimberleigh A., et Jorge Jr.VELAZQUEZ (2003). Cultural competent, A guide for human service agencies, Washington D.C, CWLA Press.

POTOCKY-TRIPODI, Miriam (2002). Best Practices for Social Work with Refugees and Immigrants, New-York, Columbia University Press.

REYNOLDS WHYTE, Susan (1995). "Disability between Discourse and Experience ", dans Disability and Culture, B. INGSTAD et S. REYNOLDS WHYTE (Dir.), Londres, California Press, p. 267-291.

SEDIGHDEILAMI, Farrokh, et Shahar GINDI (2003). "Multi cultural Issues in Assessment and Intervention for Individuals with Developmental Disabilities in Ontario ", dans Developmental disabilities in Ontario, $2^{e}$ éd., Ivan BROWN et Maire PERCY (Dir.), Ontario Association on Developmental Disabilities, p.477-492.

STATISTIQUE CANADA (2003). Enquête longitudinale auprès des immigrants du Canada : le processus, les progrès et les perspectives, Ottawa, Gouvernement du Canada.

THOMAS, Barb (1987). Multiculturalism at work : a guide to organizational change / for the YWCA of Metropolitan Toronto, Toronto, YWCA of Metropolitan Toronto.

THOMPSON, Pamela (1993). "Developing and Implementing Culturally Sensitive Health Promotion Programs ", dans Health and Cultures-Exploring the relationships, Programs, services and care, Ralph MASI, Lynette MENSAH et Keith A. McLEOD (Dir.), Oakville, Ont., Mosaic Press, Vol. 3, p. 119-252.

VICTORIAN GOVERNMENT DEPARTMENT OF HUMAN SERVICES (2004), Cultural Diversity Guide - Multicultural Strategy, State of Victoria, Australie, Department of Human Services.

WAXLER-MORRISON, Nancy (1990). "Introduction », dans Cross-cultural caring : A handbook for health professionals, Nancy WAXLER-MORRISON, Joan ANDERSON et Elisabeth RICHARDSON (Dir.), Vancouver, UBC Press, p. 3-10. 